\title{
Fluorine is Flourishing in Pharmaceuticals
}

\author{
Hua Zhang, Yu Li and Zhong-Xing Jiang*
}

School of Pharmaceutical Sciences, Wuhan University, Wuhan 430071, China

\section{Editorial}

Since Moissan isolated elemental fluorine in 1886, fluorine has always been an element of surprise. Its small size, high electron negativity and high C-F bond dissociation energy make fluorinated compounds fit perfectly in a variety of application. In the field of pharmaceuticals, introducing a fluorine atom or fluorinated group into drugs or drug leads is often accompanied with higher binding affinity, enhanced metabolic stability, improved bioavailability, and sometimes, increased selective activity. Additionally, the development and therapeutic monitoring of fluorinated drugs can be facilitated by additional techniques, such as ${ }^{18} \mathrm{~F}$ positron emission tomography (PET), ${ }^{19} \mathrm{~F}$ magnetic resonance imaging/spectroscopy (MRI/S) and fluorous technology, to name a few. To date, there are over 150 fluorinated drugs on the market which account for about $20 \%$ of all FDA approved drugs. Three out of the top 10 best selling drugs, Lipitor, Advair and Crestor, contain fluorine, and seven out of 35 newly approved drugs contain fluorine in 2011.

With recent development in novel fluorination strategies [1-8], ${ }^{18} \mathrm{~F}$ PET, ${ }^{19} \mathrm{~F}$ MRI/S and fluorous technology [9-12], fluorine is playing an ever more important role in pharmaceuticals. First, fluorination has become an established strategy to modify physical properties, binding characteristics and metabolic disposition during drug leads development. Traditionally, selective fluorination, i.e., introducing fluorine or fluorinated group at specific positions with preferred configuration, could be very challenging. Direct fluorination with fluorine gas or Diethyl Amino Sulfur Trifluoride (DAST) usually suffers low selectivity, disappointing yield, poor functional group compatibility and severe side reactions. In recent years, fluorine chemistry has become a hot spot in chemical research due to its extensive application in pharmaceuticals. Breakthroughs in this field bring about several commercially available selective fluorination reagents, such as bis(2-methoxyethyl) aminosulfur trifluoride (Deoxofluor) [1], 1-chloromethyl-4-fluorodiazoniabicyclo [2,2,2] octane bis(tetrafluoroborate) (Selectfluor) [2], and N-fluorobenzenesulfonimide (NFSI) [3]. Among the fluorinated groups, trifluoromethyl is the most useful in pharmaceuticals. Introducing trifluoromethyl group into a molecule, i.e. trifluoromethylation, can be achieved by nucleophilic approach with (trifluoromethyl)trimethylsilane (RuppertPrakash reagent) [4,5], electrophilic approach with hypervalent iodine (III) trifluoromethyl reagent (Togni's reagent [6] or Umemoto's reagent [7]), or radical approach with trifluoroiodomethane [8]. It is noteworthy that late-stage fluorination has recently been explored which allows direct modification of some chemically fragile drug leads or drug precursors [9]. Second, fluorine is also an element of choice for imaging. Two isotopes of fluorine, ${ }^{18} \mathrm{~F}$ and ${ }^{19} \mathrm{~F}$, are ideal imaging entities for tracing drugs and providing valuable pharmacodynamic and pharmacokinetic information. Information such as where the drug is, in what form, and how much, is particularly useful in both drug develop and therapy. Because of its relatively long half-life and low $\beta^{+}$-energy, ${ }^{18} \mathrm{~F}$ is the most widely used isotope for PET. ${ }^{18} \mathrm{~F}$ PET is extremely sensitive and able to trace drug at concentration as low as $10^{-9}$ $10^{-12} \mathrm{Mol}$. Therefore, it is already a widely used tool in drug biological target validation, drug biodistribution determination and rational

therapeutic dosing. On the other hand, ${ }^{19} \mathrm{~F}$ is a sensitive spin nucleus for ${ }^{19} \mathrm{~F}$ MRI/S which can provide in vivo quantitative drug information without endogenous background interference and without ionizing radiation. ${ }^{19} \mathrm{~F}$ MRS has a long history in detecting drug metabolism with its wide ${ }^{19} \mathrm{~F}$ chemical shift [10]. ${ }^{19} \mathrm{~F}$ MRI has recently been used successfully in tracing therapeutic cells [11] and drug delivery systems [12] with high spatial and temporal resolution. Such proof of concept research indicates ${ }^{19} \mathrm{~F}$ MRI is a valuable tool for imaging-guided drug delivery and personalized drug therapy. Finally, fluorous technology can dramatically simplify the purification procedures during drug synthesis [13] and rapidly identify fluorinated drugs and their metabolites during PK/PD evaluation [11].

Since nearly 70 years ago when Fried replaced $9 \alpha$-hydrogen in cortisone with fluorine and unexpectedly found single fluorine can improve bioactivity by 11 times [14], fluorine has been playing a multifaceted role in pharmaceuticals. Although fluorine was used to be regarded as an element of magic and used with intuition [15], fluorine is now used in pharmaceuticals with confidence after more details about fluorination effect on small molecule and the interaction with biological system have been revealed. Medicinal chemists are happy to see selective and efficient synthetic methods keep adding to their tool box. ${ }^{18} \mathrm{~F}$ PET, ${ }^{19} \mathrm{~F}$ MRI/S and fluorous technology give pharmacologists precious insights into drugs' in vivo behaviors and help doctors design personalized therapy. Fluorine is certain to keep surprising the pharmaceutical community.

\section{References}

1. Lal GS, Pez GP, Pesaresi RJ, Prozonic FM, Cheng H (1999) Bis(2methoxyethyl) aminosulfur trifluoride: a new broad-spectrum deoxofluorinating agent with enhanced thermal stability. J Org Chem 64: 7048-7054.

2. Singh RP, Shreeve JM (2004) Recent highlights in elelctrophilic fluorination with 1-chloromethyl-4-fluoro- 1,4-diazoniabicyclo[2,2,2]octane bis(tetrafluoroborate). Acc Chem Res 37: 31-44.

3. Differding E, Ofner H(1991) ChemInformAbstract: N-Fluorobenzenesulfonimide A Practical Reagent for Electrophilic Fluorinations. ChemInform 22.

4. Ruppert I, Schlich K, Volbach W (1984) Die ersten CF3-substituierten organyl(chlor)silane. Tetrahedron Lett. 25: 2195-2198.

5. Prakash GKS, Hu J, Olah GA (2003) Alkoxide- and hydroxide-induced nucleophilic trifluoromethylation using trifluoromethyl sulfone or sulfoxide. Org Lett 5: 3253-3256.

6. Eisenberger P, Gischig S, Togni A (2006) Novel 10-I-3 Hypervalent iodine-based compounds for electrophilic trifluoromethylation. Chem Eur J 12: 2579-2586.

*Corresponding author: Zhong-Xing Jiang, School of Pharmaceutical Sciences, Wuhan University, Wuhan 430071, China, Tel: +86-15391562217; Fax: +862768759850; E-mail: zxjiang@whu.edu.cn

Received October 30, 2012; Accepted October 31, 2012; Published November 05, 2012

Citation: Zhang H, Li Y, Jiang ZX (2012) Fluorine is Flourishing in Pharmaceuticals. J Biomol Res Ther 1:e107. doi:10.4172/2167-7956.1000e107

Copyright: ( 2012 Zhang $\mathrm{H}$, et al. This is an open-access article distributed under the terms of the Creative Commons Attribution License, which permits unrestricted use, distribution, and reproduction in any medium, provided the original author and source are credited. 
7. Umemoto T, Adachi K, Ishihara S (2007) CF oxonium salt, O-(trifluoromethyl) dibenzofuranium salt: in situ synthesis, properties, and application as a real $\mathrm{CF}_{3}+$ species reagent. J Org Chem 72: 6905-6917

8. Studer A (2012) A "renaissance" in radical trifluoromethylation. Angew Chem Int Ed Engl 51: 8950-8958

9. Capone S, Kieltsch I, Flogel O, Lelais G, Togni A, et al. (2008) Electrophillic S-trifluoromethylation of cysteine side chains in $\alpha$ - and $\beta$-peptides: isolation of trifluoromethylated Sandostatin ${ }^{\circledR}$ (Octreotide) derivatives. Helv Chim Acta 91: 2035-2056.

10. Chung YL, Troy H, Judson IR, Leek R, Leach MO, et al. (2004) Noninvasive measurements of capecitabine metabolism in bladder tumors overexpressing thymidine phosphorylase by fluorine-19 magnetic resonance spectroscopy. Clin Cancer Res 10: 3863-3870.
11. Ahrens ET, Flores $\mathrm{R}, \mathrm{Xu} \mathrm{H}$, Morel PA (2005) In vivo imaging platform for tracking immunotherapeutic cells. Nat Biotechnol. 23: 983-987.

12. Jiang ZX, Liu X, Jeong EK, Yu YB (2009) Symmetry-guided design and fluorous synthesis of a stable and rapidly excreted imaging tracer for (19)F MRI. Angew Chem Int Ed Engl 48: 4755-4758.

13. Jiang ZX, Yu YB (2010) Fluorous mixture synthesis of asymmetric dendrimers. J Org Chem 75: 2044-2049.

14. Fried J, Sabo EF (1954) 9a-Fluoro derivatives of cortisone and hydrocortisone. J Am Chem Soc 76: 1455-1456.

15. Muller K, Faeh C, Diederich F (2007) Fluorine in pharmaceuticals looking beyond intuition. Science 317: 1881-1886. 